\title{
EMBEDDING ANY COUNTABLE SEMIGROUP IN A 2-GENERATED BISIMPLE MONOID
}

\author{
by KARL BYLEEN
}

(Received 26 November, 1982)

G. B. Preston [10] proved that any semigroup can be embedded in a bisimple monoid. If $S$ is a countable semigroup, his constructive proof yields a bisimple monoid which is also countable, but not necessarily finitely generated. The main result of this paper is that any countable semigroup can be embedded in a 2-generated bisimple monoid.

J. M. Howie [6] proved that any semigroup can be embedded in an idempotentgenerated semigroup. F. Pastijn [9] showed that any semigroup can be embedded in a bisimple idempotent-generated semigroup, and that any countable semigroup can be embedded in a semigroup which is generated by 3 idempotents. Easy proofs of these results using Rees matrix semigroups over a semigroup were given by the author [3]. In this paper, as a corollary to our main result, we deduce that any countable semigroup can be embedded in a bisimple semigroup which is generated by 3 idempotents.

The proof of our main result relies on a construction of a monoid $\mathscr{C}(S ; B, A ; P)$. Given any monoid $S$, non-empty sets $A$ and $B$ which are disjoint from each other and from $S$, and an $A \times B$ matrix $P$ over $A \cup B \cup S$, a presentation is given to define $\mathscr{C}(S ; B, A ; P)$. The notation $\mathscr{C}(S ; B, A ; P)$ is chosen to reflect the nature of $\mathscr{C}(S ; B, A ; P)$ both as a generalization of the notion of a Rees matrix semigroup $\mathcal{M}(S ; B, A ; P)$ over $S$, and also as a generalization of the monoid $\mathscr{C}(S)$ which was constructed by R. H. Bruck [2] in order to show that any semigroup can be embedded in a simple monoid. Bruck's monoid $\mathscr{C}(S)$ is the monoid generated by distinct symbols $a$ and $b$ (not belonging to $S$ ) and the elements of $S$ subject to the defining relations $a b=1$, $a s=a$, $s b=b, s t=s \cdot t$ for all $s, t \in S$. An exposition of Bruck's results and alternative descriptions of $\mathscr{C}(S)$ may be found in [4]. Bruck's construction was generalized by N. R. Reilly [11] to determine the structure of all bisimple $\omega$-semigroups, and was considered in still more general form by W. D. Munn [8]. An account of these results appears in [5].

The word problem for the presentation of $\mathscr{C}(S ; B, A ; P)$ is solved in the first section of this paper. It is shown that $\mathscr{C}(S ; B, A ; P)$ contains $S$ as a submonoid, and that congruences on $S$ extend to $\mathscr{C}(S ; B, A ; P)$. In Section 2 we consider special cases in which we can elucidate the structure of $\mathscr{C}(S ; B, A ; P)$. Necessary and sufficient conditions are given on $S, B, A$, and $P$ for $\mathscr{C}(S ; B, A ; P)$ to be regular or inverse. If all entries of $P$ belong to $S$, then $\mathscr{C}(S ; B, A ; P)$ is shown to be a coextension of the bicyclic monoid by Rees matrix semigroups over $S$. In Section 3 appropriate choices of $S, B, A$ and $P$ are made to ensure that $\mathscr{C}(S ; B, A ; P)$ is a 2 -generated bisimple monoid, from which the main result is obtained. Throughout the paper the symbol $S$ is reserved to denote a monoid with identity 1 .

Glasgow Math. J. 25 (1984) 153-161. 
1. The presentation. Let $S$ be a monoid with identity 1 , let $A$ and $B$ be non-empty sets which are disjoint from each other and from $S$, and let $P=\left(p_{a b}\right)$ be an $A \times B$ matrix over $A \cup B \cup S$. Let $\mathscr{C}(S ; B, A ; P)$ denote the monoid with presentation $\langle A \cup B \cup S$; $\left.a b=p_{a b}, a s=a, s b=b, s t=s \cdot t, 1=\Lambda \forall a \in A, b \in B, s, t \in S\right\rangle$. The symbol $\Lambda$ denotes the identity (the empty word) of the free monoid $(A \cup B \cup S)^{*}$ which is generated by $A \cup B \cup S$, and $A^{*}$ and $B^{*}$ denote the free submonoids generated by $A$ and $B$, respectively. The word problem for the presentation is solved by the following lemma.

LEMMA 1.1. The elements of $\mathscr{C}(S ; B, A ; P)$ are the words in $B^{*} S A^{*}$.

Proof. The defining relations may be used to reduce any word $w \in(A \cup B \cup S)^{*}$ in a finite number of steps to a word $\bar{w} \in B^{*} S A^{*}$ by the following procedure. Let $\bar{\Lambda}=1$, $\bar{a}=1 a, \bar{b}=b 1, \bar{s}=s$. If $w=w_{1} w_{2} \ldots w_{k+1}$ has length greater than 1 , we define $\bar{w}$ by first reducing $w_{1} w_{2} \ldots w_{k}$ to the element $\overline{w_{1} w_{2} \ldots w_{k}}$ of $B^{*} S A^{*}$, and then use the equations below. Let $u, \hat{u} \in A^{*}, v \in B^{*}, s, t \in S, a \in A$, and $b \in B$.

$$
\begin{aligned}
& \overline{v t u a}=v t u a \text {; } \\
& \overline{v t u s}=\left\{\begin{array}{lll}
v(t \cdot s) & \text { if } & u=\Lambda, \\
v t u & \text { if } & u \neq \Lambda
\end{array}\right. \\
& \overline{v t u b}=\left\{\begin{array}{lll}
\frac{v b 1}{v t \hat{u} p_{a b}} & \text { if } & u=\Lambda, \\
\text { if } & u=\hat{u} a \neq \Lambda .
\end{array}\right.
\end{aligned}
$$

Note that $v t u p_{a b}$ has length less than that of $v t u b$. This inductive definition of the function $w \rightarrow \bar{w}$ establishes that any element of $(A \cup B \cup S)^{*}$ may be reduced by the defining relations to a word in $B^{*} S A^{*}$.

To complete the proof of the lemma we show that no two reduced words represent the same element of $\mathscr{C}(S ; B, A ; P)$. Let $\psi: A \cup B \cup S \rightarrow \mathscr{T}_{B^{*} S^{*}}$ be the mapping from the set $A \cup B \cup S$ into the full transformation semigroup on $B^{*} S A^{*}$ defined by $x \psi=$ $(v t u \rightarrow \overline{v t u x})$. The mapping extends to a monoid homomorphism from $(A \cup B \cup S)^{*}$ into $\mathscr{T}_{B^{*} A^{*}}$. We use the equations above to verify that each of the five types of defining relations for $\mathscr{C}(S ; B, A ; P)$ is satisfied in $\mathscr{T}_{\mathrm{B}^{*} S_{\mathrm{A}}^{*}}$ by the elements of $(A \cup B \cup S) \psi$.

(1) $(v r u)[(a \psi)(b \psi)]=(\overline{v r u a}) b \psi=($ vrua $) b \psi=\overline{\text { vruab }}=\overline{\text { vrup }}_{a b}=(v r u) p_{a b} \psi$; so $(a \psi)(b \psi)=p_{a b} \psi$

(2) $(v r u)[(a \psi)(s \psi)]=(\overline{v r u a}) s \psi=(v r u a) s \psi=\overline{\text { vruas }}=$ vrua $=\overline{\text { vrua }}=($ vru $) a \psi$; so $(a \psi)(s \psi)=a \psi$.

(3) If $u=\Lambda$ then $(v r u)[(s \psi)(b \psi)]=(\overline{v r u s}) b \psi=[v(r \cdot s)] b \psi=v b 1=\overline{v r u b}=(v r u) b \psi$. If $u \neq \Lambda$ then $(v r u)[(s \psi)(b \psi)]=(\overline{v r u s}) b \psi=(v r u) b \psi$. Thus $(s \psi)(b \psi)=b \psi$.

(4) If $u=\Lambda$ then $(v r u)[(s \psi)(t \psi)]=\overline{(v r u s)} t \psi=[v(r \cdot s)] t \psi=v((r \cdot s) \cdot t)=v(r \cdot(s \cdot t))=$ $\overline{v r u(s \cdot t)}=(v r u)[(s \cdot t) \psi]$. If $u \neq \Lambda$ then $(v r u)[(s \psi)(t \psi)]=\overline{(v r u s)} t \psi=(v r u) t \psi=\overline{v r u t}=v r u=$ $\overline{v r u(s \cdot t)}=(v r u)[(s \cdot t) \psi]$. Thus $(s \psi)(t \psi)=(s \cdot t) \psi$.

(5) If $u=\Lambda$ then $(v r u) 1 \psi=\overline{v r u 1}=v(r \cdot 1)=v r=v r u$. If $u \neq \Lambda$ then $(v r u) 1 \psi=$ $\overline{\text { vru } 1}=v r u$. Thus $1 \psi$ is the identity of $\mathscr{T}_{B^{*} S A^{*}}$.

Therefore the homomorphism $\psi$ factors through $\mathscr{C}(S ; B, A ; P)$, giving a representation of $\mathscr{C}(S ; B, A ; P)$ in $\mathscr{T}_{B^{*} S A^{*}}$. Let $w=v s u \in B^{*} S A^{*}$. If $v=\Lambda$ then $1(w \psi)=\overline{1 v s u=}$ 
$\overline{(1 \cdot s) u}=\overline{s u}=w$. If $v \neq \Lambda$ then $1(w \psi)=\overline{1 v s u}=\overline{v s u}=w$. Thus the representation is faithful (it is the right regular representation), which proves that no two reduced words represent the same element of $\mathscr{C}(S ; B, A ; P)$.

The lemma proves that each word $v s u \in B^{*} S A^{*}$ represents an element of $\mathscr{C}(S ; B, A ; P)$ and that two words $v s u$ and $v^{\prime} s^{\prime} u^{\prime}$ in $B^{*} S A^{*}$ represent the same element of $\mathscr{C}(S ; B, A ; P)$ if and only if $v=v^{\prime}, s=s^{\prime}$, and $u=u^{\prime}$. Henceforth we will denote the product in $\mathscr{C}(S ; B, A ; P)$ of two words $v s u$ and $v^{\prime} s^{\prime} u^{\prime}$ in $B^{*} S A^{*}$ simply by $v s u v^{\prime} s^{\prime} u^{\prime}$ (instead of by $\overline{v u v^{\prime} s^{\prime} u^{\prime}}$ ). We do not give an explicit formula for this product as an element of $B^{*} S A^{*}$; however, we do note that our reduction procedure implies that $v s u v^{\prime} s^{\prime} u^{\prime} \in$ $v B^{*} S A^{*} u^{\prime}$. We will find it convenient to replace 1 by $\Lambda$ in an element of $\mathscr{C}(S ; B, A ; P)$ when doing so would simplify notation; thus, for example, we will simply write $a$ or $b$ in place of $1 a$ or $b 1$, respectively.

THEOREM 1.2. The monoid $\mathscr{C}(S ; B, A ; P)$ contains $S$ as a submonoid.

Proof. The obvious mapping $\theta: S \rightarrow \mathscr{C}(S ; B, A ; P)$ defined by $s \rightarrow s$ is the required embedding since $(s \cdot t) \theta=(s \cdot t)=s t=(s \theta)(t \theta)$.

THEOREM 1.3. Any congruence on $S$ extends to a congruence on $\mathscr{C}(S ; B, A ; P)$.

Proof. Let $\phi$ be any homomorphism from the monoid $S$ onto a monoid $T$. Let $Q=\left(q_{a b}\right)$ denote the $A \times B$ matrix obtained from $P$ by replacing each entry which belongs to $S$ by its image under $\phi$ (and leaving unchanged each entry of $P$ which belongs to $A \cup B)$. Let $\alpha: A \cup B \cup S \rightarrow \mathscr{C}(T ; B, A ; Q)$ be the mapping defined by $a \rightarrow a, b \rightarrow b$, $s \rightarrow s \phi$ for all $a \in A, b \in B, s \in S$. The mapping $\alpha$ extends to a monoid homomorphism from $(A \cup B \cup S)^{*}$ into $\mathscr{C}(T ; B, A ; Q)$, also denoted by $\alpha$. We verify that the defining relations for $\mathscr{C}(S ; B, A ; P)$ are satisfied by the elements of $(A \cup B \cup S) \alpha$.

(1) $(a \alpha)(b \alpha)=a b=q_{a b}=\left\{\begin{array}{lll}p_{a b} & \text { if } & q_{a b} \in A \cup B \\ p_{a b} \phi & \text { if } & q_{a b} \in T\end{array}\right\}=p_{a b} \alpha$.

(2) $(a \alpha)(s \alpha)=a(s \phi)=a=a \alpha$.

(3) $(s \alpha)(b \alpha)=(s \phi) b=b=b \alpha$.

(4) $(s \alpha)(t \alpha)=(s \phi)(t \phi)=(s \cdot t) \phi=(s \cdot t) \alpha$.

(5) $1 \alpha=1 \phi$, the identity of $\mathscr{C}(T ; B, A ; Q)$.

Thus the homomorphism $\alpha:(A \cup B \cup S)^{*} \rightarrow \mathscr{C}(T ; B, A ; Q)$ factors through $\mathscr{C}(S ; B, A ; P)$, yielding a homomorphism $\bar{\phi}: \mathscr{C}(S ; B, A ; P) \rightarrow \mathscr{C}(T ; B, A ; Q)$ which extends $\phi$. We conclude that any congruence on $S$ extends to a congruence on $\mathscr{C}(S ; B, A ; P)$.

2. Special cases. In this section we consider several special cases of the construction of $\mathscr{C}(S ; B, A ; P)$ in which the structure of $\mathscr{C}(S ; B, A ; P)$ becomes more transparent. In this regard we note first that if the sets $A$ and $B$ are both singletons, and if the single entry of the matrix $P$ is the identity 1 of $S$ then $\mathscr{C}(S ; B, A ; P)$ is precisely the monoid $\mathscr{C}(S)$ constructed by R. H. Bruck [2].

We proceed to obtain necessary and sufficient conditions for $\mathscr{C}(S ; B, A ; P)$ to be 
regular or inverse. As a preliminary step we obtain a condition on the matrix $P$ which allows an easy description of Green's relations on $\mathscr{C}(S ; B, A ; P)$, and implies that $\mathscr{C}(S ; B, A ; P)$ is simple.

Defintrion 2.1. Let $P$ be an $A \times B$ matrix over $A \cup B \cup S$ and let $a, a^{\prime} \in A$. We say that row $a$ of $P$ is linked to row $a^{\prime}$ of $P$ if there exists a finite sequence $a=a_{1}, a_{2}, \ldots, a_{n}=$ $a^{\prime}(n \geq 1)$ such that $a_{i+1}$ appears as an entry in row $a_{i}$ of $P$ for $i=1,2, \ldots, n-1$. Similarly, for $b, b^{\prime} \in B$, we say that column $b$ of $P$ is linked to column $b^{\prime}$ of $P$ if there exists a finite sequence $b=b_{1}, b_{2}, \ldots, b_{n}=b^{\prime}(n \geq 1)$ such that $b_{i+1}$ appears as an entry in column $b_{i}$ of $P$ for $i=1,2, \ldots, n-1$.

We note that according to the definition, each row (column) of $P$ is linked to itself.

Lemma 2.2. An element $a \in A$ is right invertible in $\mathscr{b}(S ; B, A ; P)$ if and only if row a of $P$ is linked to a row which contains a right invertible element of $S$. Similarly, an element $b \in B$ is left invertible in $\mathscr{C}(S ; B, A ; P)$ if and only if column $b$ of $P$ is linked to a column which contains a left invertible element of $S$.

Proof. Suppose $a \in A$ is right invertible in $\mathscr{C}(S ; B, A ; P)$. Then there exists an element $v s u \in B^{*} S A^{*}$ such that $a(v s u)=1$. Since $a(v s u) \in B^{*} S A^{*} u$, we have $u=\Lambda$. The assertion of the lemma is proved by induction on the length $k$ of $v=b_{1} b_{2} \ldots b_{k}$. We note that $k \neq 0$ since $a s=a \neq 1$. If $k=1$ then $a b_{1} s=1$; so, since $p_{a b_{1}} \in A \cup B \cup S$, we must have $p_{a b_{1}} \in S$. Thus row $a$ itself contains an element of $S$ which is right invertible. Let $k>1$ and suppose the assertion is true whenever $v$ has length less than $k$. Since $a\left(b_{1} b_{2} \ldots b_{k}\right) s=1$, $p_{a b_{1}} \in A$. Thus, by the induction hypothesis, row $p_{a b_{1}}$ of $P$ is linked to a row, say row $a^{\prime}$, which contains a right invertible element of $S$. Since $p_{a b_{1}}$ is an entry of row $a$, row $a$ is linked to row $a^{\prime}$.

Conversely, suppose the sequence $a=a_{1}, a_{2}, \ldots, a_{k}=a^{\prime}$ links row $a$ to the row $a^{\prime}$ which contains a right invertible element of $S$. If $k=1$ then row $a$ itself contains a right invertible element, say $p_{a b}$ of $S$. In which case $a b=p_{a b}$ is right invertible; so $a$ is right invertible. Let $k>1$. By the induction hypothesis, $a_{2}$ is right invertible. But $a_{2}$ appears in row $a$, say $a_{2}=p_{a b}$. Thus $a b=a_{2}$ is right invertible; so $a$ is right invertible.

The second sentence of the lemma is true by symmetry.

Definition 2.3. An $A \times B$ matrix $P$ over $A \cup B \cup S$ is said to be united if each row of $P$ is linked to a row which contains a right invertible element of $S$ and each column is linked to a column which contains a left invertible element of $S$.

THEOREM 2.4. If $P$ is united then Green's relations on $\mathscr{C}(S ; B, A ; P)$ are as follows:

(a) $v s u \mathscr{R} v^{\prime} s^{\prime} u^{\prime} \Leftrightarrow v=v^{\prime}$ and $s \mathscr{R} s^{\prime}$ in $S$;

(b) $v s u \mathscr{L}^{\prime} v^{\prime} s^{\prime} u^{\prime} \Leftrightarrow u=u^{\prime}$ and $s \mathscr{L}^{\prime}$ in $S$;

(c) $v s u \mathscr{H} v^{\prime} s^{\prime} u^{\prime} \Leftrightarrow v=v^{\prime}, u=u^{\prime}$ and $s_{\mathscr{H}} \mathscr{H} s^{\prime}$ in $S$;

(d) $v s u \mathscr{D} v^{\prime} s^{\prime} u^{\prime} \Leftrightarrow s \mathscr{D} s^{\prime}$ in $S$;

(e) $\mathscr{J}=\omega$ and so $\mathscr{C}(S ; B, A: P)$ is simple.

Proof. (a) Suppose $v s u \mathscr{R} v^{\prime} s^{\prime} u^{\prime}$. Then there exist $x, y \in \mathscr{C}(S ; B, A ; P)$ such that 
(vsu) $x=v^{\prime} s^{\prime} u^{\prime}$ and $v s u=\left(v^{\prime} s^{\prime} u^{\prime}\right) y$. By the remarks following the proof of Lemma 1.1, these equations imply that $v$ is a prefix of $v^{\prime}$, and $v^{\prime}$ is a prefix of $v$; so $v=v^{\prime}$. Let $u x=v^{\prime \prime} s^{\prime \prime} u^{\prime \prime} \in B^{*} S A^{*}$. If $v^{\prime \prime} \neq \Lambda$ then $v s u x=v s\left(v^{\prime \prime} s^{\prime \prime} u^{\prime \prime}\right)=v v^{\prime \prime} s^{\prime \prime} u^{\prime \prime} \neq v^{\prime} s^{\prime} u^{\prime}$ since $v=v^{\prime}$. Thus $v^{\prime \prime}=\Lambda$; so $v s u x=v s\left(v^{\prime \prime} s^{\prime \prime} u^{\prime \prime}\right)=v\left(s s^{\prime \prime}\right) u^{\prime \prime}$, and hence $s s^{\prime \prime}=s^{\prime}$; so $s^{\prime} \in S S$. Similarly $s \in s^{\prime} S$; so $s \mathscr{R} s^{\prime}$ in $S$. (We note that the assumption that $P$ is united has not been used for this half of the result.) Conversely, suppose $v=v^{\prime}$ and.s $\mathscr{R} s^{\prime}$ in $S$. Then there exist $t, t^{\prime} \in S$ such that $s t=s^{\prime}, s=s^{\prime} t^{\prime}$. Since $P$ is united it follows from Lemma 2.2 that $u$ and $u^{\prime}$ are right invertible in $\mathscr{C}(S ; B, A ; P)$, say $u x=1, u^{\prime} x^{\prime}=1$. Thus $v s u\left(x t u^{\prime}\right)=v s t u^{\prime}=v s^{\prime} u^{\prime}=v^{\prime} s^{\prime} u^{\prime}$ and $v^{\prime} s^{\prime} u^{\prime}\left(x^{\prime} t^{\prime} u\right)=v^{\prime} s^{\prime} t^{\prime} u=v^{\prime} s u=v s u$; so $v s u \mathscr{R} v^{\prime} s^{\prime} u^{\prime}$.

(b) The proof is entirely similar to that of (a).

(c) and (d) follow from (a) and (b).

(e) Let $v s u \in \mathscr{C}(S ; B, A ; P)$. Since $P$ is united it follows from Lemma 2.2 that $v$ is left invertible and $u$ is right invertible in $\mathscr{C}(S ; B, A ; P)$, say $x v=1$, $u y=1$. Let $a \in A$. Since $a$ is right invertible, there exists $z \in \mathscr{C}(S ; B, A ; P)$ such that $a z=1$. Therefore $(a x)(v s u)(y z)=a s z=a z=1$; so 1 belongs to the ideal generated by vsu. Hence $\mathscr{C}(S ; B, A ; P)$ is simple.

Corollary 2.5. Let $P$ be united. Then $\mathscr{C}(S ; B, A ; P)$ is bisimple if and only if $S$ is bisimple.

THEOREM 2.6. The monoid $\mathscr{C}(S ; B, A ; P)$ is regular if and only if $S$ is regular and $P$ is united.

Proof. Suppose $\mathscr{C}(S ; B, A ; P)$ is regular. Let $s \in S$. Then there exists $v t u \in B^{*} S A^{*}$ such that $s(v t u) s=s$. Hence $v=\Lambda=u$; so $S$ is regular. Let $a \in A$. Then there exists $v t u \in B^{*} S A^{*}$ such that $a(v t u) a=a$. By the remarks following the proof of Lemma 1.1, $a(v t u) a \in B^{*} S A^{*} a$; so $a(v t u)=1$. Thus $a$ is right invertible; so, by Lemma 2.2 , row $a$ of $P$ is linked to a row which contains a right invertible element of $S$. The argument is symmetrical for columns of $P$. Hence $P$ is united.

Conversely, assume the conditions of the theorem hold, and let $v s u \in B^{*} S A^{*}$. Since $S$ is regular, there exists $t \in S$ such that $s t s=s$. By the use of Lemma 2.2, there exist $x, y \in \mathscr{C}(S ; B, A ; P)$ such that $u x=1=y v$. Thus $v s u(x t y) v s u=v s u$; so $v s u$ is regular. Hence $\mathscr{C}(S ; B, A ; P)$ is regular.

THEOREM 2.7. The monoid $\mathscr{C}(S ; B, A ; P)$ is inverse if and only if $S$ is inverse, $A$ and $B$ are singletons, and the single entry of $P$ is an invertible element of $S$.

Proof. Suppose $\mathscr{C}(S ; B, A ; P)$ is inverse. Then $S$, being a regular submonoid of $\mathscr{C}(S ; B, A ; P)$ by Theorem 2.6 , is inverse. Let $a_{1}, a_{2} \in A$. Since $a_{1}$ and $a_{2}$ are right invertible there exist $x_{1}, x_{2} \in \mathscr{C}(S ; B, A ; P)$ such that $a_{1} x_{1}=1, a_{2} x_{2}=1$. Thus $x_{1} a_{1}$ and $x_{2} a_{2}$ are idempotents. Since $\mathscr{C}(S ; B, A ; P)$ is inverse, $x_{1} a_{1} x_{2} a_{2}=x_{2} a_{2} x_{1} a_{1}$. But $x_{1} a_{1} x_{2} a_{2} \in$ $\left(B^{*} S A^{*}\right) a_{2}$ and $x_{2} a_{2} x_{1} a_{1} \in\left(B^{*} S A^{*}\right) a_{1}$; so $a_{1}=a_{2}$. Hence $A$, and similarly $B$, is a singleton. By Theorem 2.6, the matrix $P$ is united, and thus the single entry of $P$ must be an invertible element of $S$.

Conversely, if $S$ is inverse, $A$ and $B$ are singletons, and the single entry $g$ of $P$ is an 
invertible element of $S$ then the elements of $\mathscr{C}(S ; B, A ; P)$ are $b^{n} s a^{m}, s \in S, n, m=$ $0,1,2, \ldots$ Since $a b=g \in S$ we have $a^{2} b^{2}=a g b=a b$. Thus the idempotents of $\mathscr{C}(S ; B, A ; P)$ are the idempotents of $S$ together with all elements $b^{n} s a^{n}(n \geq 1)$ for which $s g s=s$. We claim that any two idempotents of $\mathscr{C}(S ; B, A ; P)$ commute. Let $s, t \in S$ with $s g s=s, \operatorname{tg} t=t$. Since idempotents commute in $S$, we have $s g t=s g t g t=\operatorname{tgsg} t=\operatorname{tg} \operatorname{tg} s=\operatorname{tgs}$. Thus $b^{n} s a^{n} \cdot b^{n} t a^{n}=b^{n} s g t a^{n}=b^{n} t g s a^{n}=b^{n} t a^{n} \cdot b^{n} s a^{n}$ for $n \geq 1$. If $1 \leq n<m$ then $b^{m} s a^{m} \cdot b^{n} t a^{n}=b^{m} s a^{m}=b^{n} t a^{n} \cdot b^{m} s a^{m}$. If $e^{2}=e \in S$ then $e \cdot b^{n} s a^{n}=b^{n} s a^{n}=b^{n} s a^{n} \cdot e$. Therefore idempotents commute in the regular monoid $\mathscr{C}(S ; B, A ; P)$ and so $\mathscr{C}(S ; B, A ; P)$ is inverse.

We have not given an explicit formula for the product of two elements of $\mathscr{C}(S ; B, A ; P)$ in general. However, in the special case in which all entries of the matrix $P$ belong to $S$, we can give such a formula. Let $v s u, w t x \in B^{*} S A^{*}$ and let $u=u_{1} u_{2} \ldots u_{n}$, $w=w_{m} w_{m-1} \ldots w_{1}$ (if $u=\Lambda$ or $w=\Lambda$, let $n=0$ or $m=0$, respectively). Then

$$
v s u \cdot w t x=\left\{\begin{array}{lll}
v s\left(u_{1} u_{2} \ldots u_{n-m} x\right) & \text { if } & n>m, \\
v(s t) x & \text { if } & n=m=0, \\
v\left(s p_{u_{1} w_{1}} t\right) x & \text { if } & n=m>0, \\
\left(v w_{m-n} \ldots w_{2} w_{1}\right) t x & \text { if } & n<m .
\end{array}\right.
$$

In this case $B S A$ is clearly a subsemigroup of $\mathscr{C}(S ; B, A ; P)$ which is isomorphic to the Rees matrix semigroup $\mathcal{M}(S ; B, A ; P)$. Furthermore, we show that there exists a homomorphism from $\mathscr{C}(S ; B, A ; P)$ onto the bicyclic monoid such that pre-images of idempotents are isomorphic to Rees matrix semigroups over $S$.

THEOREM 2.8. If each entry of $P$ belongs to $S$ then the monoid $\mathscr{C}(S ; B, A ; P)$ is a coextension of the bicyclic monoid by Rees matrix semigroups over $S$.

Proof. Let $\phi: A \cup B \cup S \rightarrow \mathscr{C}(p, q)$ be the mapping into the bicyclic monoid such that $a \rightarrow p, b \rightarrow q, s \rightarrow 1$ for all $a \in A, b \in B, s \in S$. Then each of the five types of defining relations for $\mathscr{C}(S ; B, A ; P)$ is clearly satisfied by the elements of $(A \cup B \cup S) \phi$; so $\phi$ factors through $\mathscr{C}(S ; B, A ; P)$, yielding a homomorphism from $\mathscr{C}(S ; B, A ; P)$ onto $\mathscr{C}(p, q)$, also denoted by $\phi$. Thus $(v s u) \phi=q^{m} p^{n}$ for $v s u \in B^{*} S A^{*}$, where $m$ is the length of $v$ and $n$ is the length of $u$. Therefore the idempotent $q^{n} p^{n}$ of $\mathscr{C}(p, q)$ has pre-image $B^{n} S A^{n}$. In particular, $\phi^{-1}(1)=S$. From the multiplication in $\mathscr{C}(S ; B, A ; P)$ it follows that for $n \geq 1, B^{n} S A^{n}$ is isomorphic to the Rees matrix semigroup $M\left(S ; B^{n}, A^{n} ; P(n)\right)$ over $S$, where $P(n)=\left(p(n)_{u v}\right)$ is the $A^{n} \times B^{n}$ matrix over $S$ defined by $p(n)_{u v}=p_{u_{1} v_{1}}$ for $u=$ $u_{1} u_{2} \ldots u_{n}, v=v_{n} v_{n-1} \ldots v_{1}$. If $n=1$ then $P(n)$ equals $P$, and $B S A=\mathcal{M}(S ; B, A ; P)$. If $n>1$ then $P(n)$ may be viewed as an $A \times B$ matrix of blocks $P(n)_{a b}$, where each block $P(n)_{a b}$ is a constant $A^{n-1} \times B^{n-1}$ matrix having value $p_{a b}$. We have shown that the pre-image of each idempotent is isomorphic to a Rees matrix semigroup over $S$, i.e. $\mathscr{C}(S ; B, A ; P)$ is a coextension of $\mathscr{C}(p, q)$ by Rees matrix semigroups over $S$.

THEOREM 2.9. If $S$ is regular and all entries of $P$ belong to $S$ then the regular elements of $\mathscr{C}(S ; B, A ; P)$ form a regular submonoid of $\mathscr{C}(S ; B, A ; P)$. 
Proof. Let $v s u$ and $w t x$ be regular elements of $\mathscr{C}(S ; B, A ; P)$, where $u=u_{1} u_{2} \ldots u_{n}$ and $w=w_{m} w_{m-1} \ldots w_{1}$ (if $u=\Lambda$ or $w=\Lambda$, let $n=0$ or $m=0$ as above). Then there exist elements $y, z \in \mathscr{C}(S ; B, A ; P)$ such that $(v s u) y(v s u)=v s u$ and $(w t x) z(w t x)=w t x$. By the remarks following the proof of Lemma 1.1, we have suyvs $=s$ and $t x z w t=t$.

Case 1. Suppose $n>m$. Let $k=z w t u_{n-m+1} \ldots u_{n} y$. Then

$$
\begin{aligned}
(v s u w t x) k(v s u w t x) & =\left(v s u_{1} u_{2} \ldots u_{n-m} x\right) k(v s u w t x) \\
& =\left(v s u_{1} u_{2} \ldots u_{n-m} t x\right) z w t u_{n-m+1} \ldots u_{n} y(v s u w t x) \\
& =v s u y v s u w t x \\
& =v s u w t x
\end{aligned}
$$

so vsuwtx is regular.

Case 2. Suppose $n=m=0$. Since $S$ is regular, there exists $r \in S$ such that $(s t) r(s t)=$ st. Let $k=z w$ trsuy. Then

$$
\begin{aligned}
(v s u w t x) k(v s u w t x) & =(v s t x) k(v s t x) \\
& =(v s t x) z w t r s u y(v s t x) \\
& =v s t r s t x \\
& =v s t x \\
& =v s u w t x
\end{aligned}
$$

so vsuwtx is regular.

Case 3. Suppose $n=m>0$. Since $S$ is regular and $p_{u_{1} w_{1}} \in S$, there exists $r \in S$ such that $\left(s p_{u_{1} w_{1}} t\right) r\left(s p_{u_{1} w_{1}} t\right)=s p_{u_{1} w_{1}} t$. Let $k=z w t r s u y$. Then

$$
\begin{aligned}
(v s u w t x) k(v s u w t x) & =\left(v s p_{u_{1} w_{1}} t x\right) z w t r s u y\left(v s p_{u_{1} w_{1}} t x\right) \\
& =v s p_{u_{1} w_{1}} t r p_{u_{1} w_{1}} t x \\
& =v s p_{u_{1} w_{1}} t x \\
& =v s u w t x
\end{aligned}
$$

so vsuwtx is regular.

Case 4. Suppose $n<m$. Let $k=z w_{m} \ldots w_{m-n+1}$ suy. Then

$$
\begin{aligned}
(v s u w t x) k(v s u w t x) & =(v s u w t x) k\left(v w_{m-n} \ldots w_{2} w_{1} t x\right) \\
& =(v s u w t x) z w_{m} \ldots w_{m-n+1} s u y v s w_{m-n} \ldots w_{2} w_{1} t x \\
& =(v s u w t x) z(w t x) \\
& =v s u w t x
\end{aligned}
$$

so vsuwtx is regular.

We conclude that the regular elements of $\mathscr{C}(S ; B, A ; P)$ form a regular submonoid. 
We note that the observation of D. B. McAlister [7], that the regular elements of any Rees matrix semigroup over a regular semigroup form a subsemigroup, follows easily from Theorems 2.8 and 2.9 .

3. The embedding theorem. The main result is an application of the construction of the monoid $\mathscr{C}(S ; B, A ; P)$.

THEOREM 3.1. Any countable semigroup can be embedded in a 2-generated bisimple monoid.

Proof. By the result of Preston [10] referred to in the introduction, any countable semigroup can be embedded in a countable bisimple monoid. So it suffices to prove the theorem for any countable bisimple monoid $S$. Let $A=\left\{a_{1}, a_{2}, a_{3}, \ldots\right\}$ and $B=$ $\left\{b_{1}, b_{2}, b_{3}, \ldots\right\}$ be countably infinite sets, disjoint from each other and from $S$. Let $P$ be an $A \times B$ matrix over $A \cup B \cup S$ such that (1) $p_{n n}=a_{n+1}$ and $p_{n+1, n}=b_{n+1}$ for $n=$ $1,2,3, \ldots$, (2) those entries of $P$ which belong to $S$ generate $S$, and (3) $P$ is united. These conditions can be satisfied, for example, by placing the identity 1 of $S$ in each row and column of $P$, and by placing each element of $S$ somewhere in $P$. By Corollary 2.5 , $\mathscr{C}(S ; B, A ; P)$ is bisimple. Since $a_{n} b_{n}=p_{n n}=a_{n+1}$ and $a_{n+1} b_{n}=p_{n+1, n}=b_{n+1}$ for $n=$ $1,2,3, \ldots$, each element of $A \cup B$ is generated by $a_{1}$ and $b_{1}$. Furthermore, since each element of $S$ is generated by elements of the form $a_{n} b_{m}=p_{n m}, A \cup B \cup S$ and thus $\mathscr{C}(S ; B, A ; P)$ is generated by the elements $a_{1}$ and $b_{1}$.

CoRollary 3.2. Any countable semigroup can be embedded in a bisimple semigroup which is generated by 3 idempotents.

Proof. By Theorem 3.1, any countable semigroup can be embedded in a bisimple monoid $\mathscr{C}(S ; B, A ; P)$ which is generated by elements $a_{1}$ and $b_{1}$. The Rees matrix semigroup $\mathcal{M}(\mathscr{C}(S ; B, A ; P) ; 3,3 ; Q)$ over $\mathscr{C}(S ; B, A ; P)$, where

$$
Q=\left[\begin{array}{ccc}
1 & 1 & 1 \\
1 & 1 & a_{1} \\
1 & b_{1} & 1
\end{array}\right],
$$

is bisimple and generated by the 3 idempotents $(1,1,1),(2,1,2),(3,1,3)$.

We note that the numbers of generators in Theorem 3.1 and Corollary 3.2 are the best possible since any bisimple monoid generated by a single element is a finite cyclic group, and since any bisimple semigroup generated by 2 idempotents is completely simple. Semigroups which are generated by 2 idempotents have been completely classified by Benzaken and Mayr [1]. Our results indicate that semigroups generated by 3 idempotents, even those which are bisimple, can be very complicated.

\section{REFERENCES}

1. C. Benzaken and H. C. Mayr, Notion de demi-bande: demi-bandes de type deux, Semigroup Forum 10 (1975), 115-128.

2. R. H. Bruck, A survey of binary systems, Ergebnisse der Math. Heft 20 (Springer, 1958). 
3. K. Byleen, Regular four-spiral semigroups, idempotent-generated semigroups, and the Rees construction, Semigroup Forum 22 (1981), 97-100.

4. A. H. Clifford and G. B. Preston, The algebraic theory of semigroups (Amer. Math. Soc. 1961, 1967).

5. J. M. Howie, An introduction to semigroup theory (Academic Press, 1976).

6. J. M. Howie, The subsemigroup generated by the idempotents of a full transformation semigroup, J. London Math. Soc. 41 (1966), 707-716.

7. D. B. McAlister, Regular Rees matrix semigroups and regular Dubreil-Jacotin semigroups, J. Austral. Math. Soc. Ser. A 31 (1981), 325-336.

8. W. D. Munn, On simple inverse semigroups, Semigroup Forum 1 (1970), 63-74.

9. F. Pastijn, Embedding semigroups in semibands, Semigroup Forum 14 (1977), 247-263.

10. G. B. Preston, Embedding any semigroup in a $\mathscr{D}$-simple semigroup, Trans. Amer. Math. Soc. 93 (1959), 351-355.

11. N. R. Reilly, Bisimple $\omega$-semigroups, Proc. Glasgow Math. Assoc. 7 (1966), 160-167.

Department of Mathematics, Statistics, and Computer Science

MARQueTtE UnIVERSTTY

MiLWAUKeE, WisCONSIN 53233 